\title{
ON A DIRICHLET PROBLEM WITH $p$-LAPLACIAN AND SET-VALUED NONLINEARITY
}

\author{
S. A. MARANO
}

(Received 19 August 2011)

Abstract

The existence of solutions to a homogeneous Dirichlet problem for a $p$-Laplacian differential inclusion is studied via a fixed-point type theorem concerning operator inclusions in Banach spaces. Some meaningful special cases are then worked out.

2010 Mathematics subject classification: primary 35J60; secondary 35R70, 47H15.

Keywords and phrases: p-Laplacian, differential inclusion, generalized gradient, operator inclusion.

\section{Introduction}

Let $\Omega$ be a bounded domain in $\mathbb{R}^{N}$ with a smooth boundary $\partial \Omega$, let $p \in(1,+\infty)$, and let $j: \Omega \times \mathbb{R} \rightarrow \mathbb{R}$ be measurable in $x \in \Omega$ for every $z \in \mathbb{R}$. Consider the Dirichlet problem

$$
\begin{cases}-\Delta_{p} u=j(x, u) & \text { in } \Omega, \\ u=0 & \text { on } \partial \Omega,\end{cases}
$$

where $\Delta_{p} u:=\operatorname{div}\left(|\nabla u|^{p-2} \nabla u\right)$ denotes the $p$-Laplacian. If $j$ is a Carathéodory's function then a number of existence and multiplicity results involving (1.1) are available in the literature; see for instance the monographs [8, 9, 15], besides the very recent paper [3]. Variational, subsupersolutions, as well as topological methods represent the most exploited technical approaches. When $j(x, \cdot)$ turns out to be locally essentially bounded only, (1.1) is usually replaced by

$$
\begin{cases}-\Delta_{p} u \in \partial J(x, u) & \text { in } \Omega, \\ u=0 & \text { on } \partial \Omega,\end{cases}
$$

with

$$
J(x, \xi):=\int_{0}^{\xi} j(x, t) d t, \quad(x, \xi) \in \Omega \times \mathbb{R}
$$

(C) 2011 Australian Mathematical Publishing Association Inc. 0004-9727/2011 \$16.00 
and $\partial J(x, z)$ being the Clarke generalized gradient of $J(x, \cdot)$ at the point $z \in \mathbb{R}$. Problem (1.2) has been the subject of numerous investigations, mainly based on the critical point theory for locally Lipschitz continuous functions [4, 10, 14], sometimes combined with subsupersolution arguments $[2,8]$. By the way, setting

$$
\underline{j}(x, z):=\lim _{\delta \rightarrow 0^{+}} \underset{|w-z|<\delta}{\operatorname{essinf}} j(x, w), \quad \bar{j}(x, z):=\lim _{\delta \rightarrow 0^{+}} \underset{|w-z|<\delta}{\operatorname{ess} \sup } j(x, w), \quad(x, z) \in \Omega \times \mathbb{R},
$$

the inclusion in (1.2) becomes

$$
\underline{j}(x, u) \leq-\Delta_{p} u \leq \bar{j}(x, u) \quad \text { in } \Omega,
$$

which reduces to $-\Delta_{p} u=j(x, u)$ at each point $u$ where $j(x, \cdot)$ is continuous.

In this paper, we simply point out that Problem (1.2), with $J$ unnecessarily of the type (1.3), can also be treated through an existence result for operator inclusions, previously established in [1], provided $p>N$. One assumes that $(x, z) \mapsto J(x, z)$, $(x, z) \in \Omega \times \mathbb{R}$, is measurable with respect to $x \in \Omega$ and locally Lipschitz continuous in $z \in \mathbb{R}$. A further condition, compatible with any growth rate of $J(x, \cdot)$, fits our purposes; see Theorem 3.1. Some meaningful special cases, namely Corollaries 3.2-3.3, are then worked out.

The recent work [7] treats $p$-Laplacian differential inclusions via fixed points for multifunctions in partially ordered sets. Amidst the results of [7] let us mention Proposition 4.1, which provides extremal solutions to a problem like (1.5) under hypotheses different from those employed here.

\section{Preliminary results}

From now on, $\Omega$ denotes a bounded domain of the real Euclidean $N$-space $\left(\mathbb{R}^{N},|\cdot|\right)$ with a smooth boundary $\partial \Omega, p \in(N,+\infty), p^{\prime}:=p /(p-1),\|\cdot\|_{q}$ is the usual norm of $L^{q}(\Omega), 1 \leq q \leq+\infty$, while $W_{0}^{1, p}(\Omega)$ stands for the closure of $C_{0}^{\infty}(\Omega)$ in $W^{1, p}(\Omega)$. On $W_{0}^{1, p}(\Omega)$ we introduce the norm

$$
\|u\|:=\left(\int_{\Omega}|\nabla u(x)|^{p} d x\right)^{1 / p}, \quad u \in W_{0}^{1, p}(\Omega) .
$$

It is known that $W_{0}^{1, p}(\Omega)$ compactly embeds in $L^{p}(\Omega)$ and one has

$$
\|u\|_{p} \leq \lambda_{1}^{-1 / p}\|u\| \quad \forall u \in W_{0}^{1, p}(\Omega)
$$

where $\lambda_{1}$ indicates the first Dirichlet eigenvalue of the $p$-Laplacian [11]. Moreover, since $p>N$, we actually get $W_{0}^{1, p}(\Omega) \subseteq L^{\infty}(\Omega)$ as well as

$$
\|u\|_{\infty} \leq a\|u\|, \quad u \in W_{0}^{1, p}(\Omega),
$$

for suitable $a>0$; see, for example, [5, Ch. IX]. The constant $a$ has been estimated in [16, Formula (6b)] and, for convex $\Omega$, in [6, Theorem 1]. 
Let $W^{-1, p^{\prime}}(\Omega)$ be the dual space of $W_{0}^{1, p}(\Omega)$. By [5, Theorem VI.4] the space $L^{p^{\prime}}(\Omega)$ compactly embeds in $W^{-1, p^{\prime}}(\Omega)$. Thus, there exists $b>0$ satisfying

$$
\|v\|_{W^{-1, p^{\prime}}(\Omega)} \leq b\|v\|_{p^{\prime}}, \quad v \in L^{p^{\prime}}(\Omega) .
$$

REMARK 2.1. The constant $b$ can be evaluated through $\lambda_{1}$. In fact,

$$
\|v\|_{W^{-1, p^{\prime}}(\Omega)}:=\sup _{\|u\| \leq 1}\left|\int_{\Omega} u(x) v(x) d x\right| \leq \sup _{\|u\| \leq 1}\|u\|_{p}\|v\|_{p^{\prime}} \leq \lambda_{1}^{-1 / p}\|v\|_{p^{\prime}}
$$

for all $v \in L^{p^{\prime}}(\Omega)$, whence $b \leq \lambda_{1}^{-1 / p}$.

Let $A: W_{0}^{1, p}(\Omega) \rightarrow W^{-1, p^{\prime}}(\Omega)$ be the nonlinear operator stemming from the negative $p$-Laplacian, that is,

$$
\langle A(u), v\rangle:=\int_{\Omega}|\nabla u(x)|^{p-2} \nabla u(x) \cdot \nabla v(x) d x, \quad u, v \in W_{0}^{1, p}(\Omega) .
$$

Theorem A.0.6 in [15] and an elementary argument ensure the following properties.

$\left(\mathrm{p}_{1}\right) A$ is bijective and uniformly continuous on bounded sets.

$\left(\mathrm{p}_{2}\right)$ Its inverse $A^{-1}$ turns out to be continuous.

( $\left.\mathrm{p}_{3}\right)\|A(u)\|_{W^{-1, p^{\prime}}(\Omega)}=\|u\|^{p-1}$ in $W_{0}^{1, p}(\Omega)$.

Let $U$ be a nonempty set and let $\Phi: U \rightarrow W_{0}^{1, p}(\Omega), \Psi: U \rightarrow L^{p^{\prime}}(\Omega)$ be two operators such that the following conditions $\left(\mathrm{i}_{1}\right)$ hold true.

$\left(i_{1}\right) \quad \Psi$ is bijective and for any $v_{h} \rightarrow v$ in $L^{p^{\prime}}(\Omega)$ there is a subsequence of $\left\{\Phi\left(\Psi^{-1}\left(v_{h}\right)\right)\right\}$ which converges to $\Phi\left(\Psi^{-1}(v)\right)$ almost everywhere in $\Omega$. Furthermore, a nondecreasing function $\varphi: \mathbb{R}_{0}^{+} \rightarrow \mathbb{R}_{0}^{+} \cup\{+\infty\}$ can be defined in such a way that

$$
\|\Phi(u)\|_{\infty} \leq \varphi\left(\|\Psi(u)\|_{p^{\prime}}\right) \quad \forall u \in U .
$$

Finally, let $F: \Omega \times \mathbb{R} \rightarrow 2^{\mathbb{R}}$ be a convex closed-valued multifunction. Theorem 3.1 of [1] directly yields the next result.

Theorem 2.2. Suppose $\left(i_{1}\right)$ holds true and, moreover, suppose that the following conditions hold true.

(i $\left.\mathrm{i}_{2}\right) \quad F(\cdot, z)$ is measurable for all $z \in \mathbb{R}$.

$\left(\mathrm{i}_{3}\right) \quad F(x, \cdot)$ has a closed graph for almost every $x \in \Omega$.

$\left(_{4}\right)$ There exists $r>0$ such that the function $m(x):=\sup _{|z| \leq \varphi(r)} \inf \{|y|: y \in F(x, z)\}$, $x \in \Omega$, belongs to $L^{p^{\prime}}(\Omega)$ and $\|m\|_{p^{\prime}} \leq r$.

Then the problem $\Psi(u) \in F(x, \Phi(u))$ in $\Omega$ possesses at least one solution $u \in U$ satisfying $|\Psi(u)(x)| \leq m(x)$ for almost every $x \in \Omega$.

For the notions on multifunctions (respectively, nonsmooth analysis) exploited in the paper, we simply refer the reader to [1] (respectively, [12]), measurable always means Lebesgue measurable, while the symbol $m(E)$ will indicate the Lebesgue measure of $E$. 


\section{Existence of solutions}

Keep the same notation of Section 2 and define, for every $t \in \mathbb{R}_{0}^{+}$,

$$
\varphi(t):=a(b t)^{1 /(p-1)} .
$$

The function $\varphi$ turns out to be monotone increasing in $\mathbb{R}_{0}^{+}$. Let $J: \Omega \times \mathbb{R} \rightarrow \mathbb{R}$. We shall make the following assumptions.

(a $\left.\mathrm{a}_{1}\right)(\cdot, z), z \in \mathbb{R}$, is measurable.

$\left(\mathrm{a}_{2}\right)$ To every $M>0$ there corresponds $k(M)>0$ such that

$$
\begin{array}{cl}
\left|J\left(x, z_{1}\right)-J\left(x, z_{2}\right)\right| \leq k(M)\left|z_{1}-z_{2}\right| & \text { almost everywhere in } \Omega \text { and } \\
& \forall z_{1}, z_{2} \in[-M, M] .
\end{array}
$$

(a) For suitable $\varepsilon, r>0$ one has $m(\Omega)^{1-1 / p} k\left(a(b r)^{1 /(p-1)}+\varepsilon\right) \leq r$.

By $\left(\mathrm{a}_{2}\right)$ it makes sense to consider the generalized Clarke gradient $\partial J(x, z)$ of $J(x, \cdot)$ at the point $z \in \mathbb{R}$.

THEOREM 3.1. If $p>N$ and $\left(a_{1}\right)-\left(a_{3}\right)$ hold true then there exists $u \in W_{0}^{1, p}(\Omega)$ satisfying $-\Delta_{p} u(x) \in \partial J(x, u(x))$ almost everywhere in $\Omega$.

Proof. Set $U:=A^{-1}\left(L^{p^{\prime}}(\Omega)\right), \Phi(u):=u$, and $\Psi(u):=A(u)$ for all $u \in U$. Property $\left(\mathrm{p}_{1}\right)$ ensures that the operator $\Psi: U \rightarrow L^{p^{\prime}}(\Omega)$ is bijective. Let $v_{h} \rightarrow v$ in $L^{p^{\prime}}(\Omega)$. Because of the compact embedding $L^{p^{\prime}}(\Omega) \subseteq W^{-1, p^{\prime}}(\Omega)$ and $\left(\mathrm{p}_{2}\right)$ we obtain, up to subsequences, $\Phi\left(\Psi^{-1}\left(v_{h}\right)\right) \rightarrow \Phi\left(\Psi^{-1}(v)\right)$ almost everywhere in $\Omega$. Hence, $\left(i_{1}\right)$ is verified once we prove (2.4). Since $p>N$, gathering (2.1), (2.2), and ( $\left.\mathrm{p}_{3}\right)$ together, one has

$$
\|\Phi(u)\|_{\infty} \leq a\|u\|=a\|\Psi(u)\|_{W^{-1, p^{\prime}(\Omega)}}^{1 /(p-1)} \leq a\left(b\|\Psi(u)\|_{p^{\prime}}\right)^{1 /(p-1)}=\varphi\left(\|\Psi(u)\|_{p^{\prime}}\right), \quad u \in U,
$$

with $\varphi$ given by (3.1), and $\left(i_{1}\right)$ follows.

Now define $F(x, z):=\partial J(x, z),(x, z) \in \Omega \times \mathbb{R}$. A simple computation shows that

$$
F(x, z)=\left[-J^{0}(x, z ;-1), J^{0}(x, z ;+1)\right],
$$

where, as usual,

$$
J^{0}(x, z ; \pm 1):=\limsup _{w \rightarrow z, t \rightarrow 0^{+}} \frac{J(x, w \pm t)-J(x, w)}{t} .
$$

Thanks to $\left(\mathrm{a}_{1}\right)$ the functions $x \mapsto J^{0}(x, z ; \pm 1)$ are measurable in $\Omega$ for every $z \in \mathbb{R}$. So, taking account of [13, Proposition 1.1], condition $\left(i_{2}\right)$ of Theorem 2.2 holds.

Let us next verify $\left(\mathrm{i}_{3}\right)$. Pick $\left\{z_{h}\right\},\left\{y_{h}\right\} \subseteq \mathbb{R}$ fulfilling

$$
z_{h} \rightarrow z, \quad y_{h} \rightarrow y, \quad y_{h} \in F\left(x, z_{h}\right) \quad \forall h \in \mathbb{N} .
$$

The upper semicontinuity of $\zeta \mapsto J^{0}(x, \zeta ; \pm 1)$, combined with (3.2), yield, as $h \rightarrow+\infty$,

$$
-J^{0}(x, z ;-1) \leq y \leq J^{0}(x, z ;+1), \quad \text { namely } y \in F(x, z),
$$

which represents the desired conclusion. 
Finally, to prove $\left(i_{4}\right)$ observe at first that

$$
\left|J^{0}(x, z ; \pm 1)\right| \leq k(M) \quad \forall M>0, z \in(-M, M) .
$$

This implies

$$
m(x):=\sup _{|z| \leq \varphi(r)} \inf \{|y|: y \in F(x, z)\} \leq \sup _{|z|<\varphi(r)+\epsilon} \inf \{|y|: y \in F(x, z)\} \leq k(\varphi(r)+\epsilon)
$$

almost everywhere in $\Omega$. Consequently, by $\left(\mathrm{a}_{3}\right)$,

$$
\|m\|_{p^{\prime}} \leq m(\Omega)^{1-1 / p} k(\varphi(r)+\epsilon) \leq r .
$$

Now Theorem 2.2 can be applied, and we obtain $u \in U \subseteq W_{0}^{1, p}(\Omega)$ such that

$$
-\Delta_{p} u(x)=\Psi(u)(x) \in F(x, u(x))=\partial J(x, u(x))
$$

for almost all $x \in \Omega$.

A meaningful special case occurs when $J$ is given by (1.3), where $j: \Omega \times \mathbb{R} \rightarrow \mathbb{R}$ fulfils the following hypotheses.

(a) $j$ turns out to be measurable in each variable separately.

(a) To every $M>0$ there corresponds $k(M)>0$ such that $|j(x, z)| \leq k(M)$ almost everywhere in $\Omega$ and for all $z \in[-M, M]$.

Indeed, under $\left(\mathrm{a}_{4}\right)-\left(\mathrm{a}_{5}\right)$, the function $J$ satisfies $\left(\mathrm{a}_{1}\right),\left(\mathrm{a}_{2}\right)$, and we get

$$
\partial J(x, z)=[\underline{j}(x, z), \bar{j}(x, z)],
$$

with $j, \bar{j}$ being as in (1.4); see [12, Example 1]. Hence, Theorem 3.1 directly leads to the following corollary.

Corollary 3.2. If $\left(a_{4}\right)-\left(a_{5}\right)$, besides $\left(a_{3}\right)$, hold true then there exists $u \in W_{0}^{1, p}(\Omega)$ such that $\underline{j}(x, u(x)) \leq-\Delta_{p} u(x) \leq \bar{j}(x, u(x))$ for almost every $x \in \Omega$.

In particular, when

$$
|j(x, z)| \leq c_{1}+c_{2}|z|^{p-1} \quad \forall(x, z) \in \Omega \times \mathbb{R},
$$

where $c_{1}, c_{2}>0$, from the above result we deduce the following corollary.

Corollary 3.3. Let the function $j$ comply with $\left(a_{4}\right)$ and (3.3). Assume also that

$$
m(\Omega)^{1-1 / p} a^{p-1} b c_{2}<1 .
$$

Then the conclusion of Corollary 3.2 holds. 
Remark 3.4. Applications of Theorem 3.1 and its consequences can basically be constructed only if one knows explicit estimates of constants $a$ and $b$. As already observed in Section 2, thanks to [16, Formula (6b)] we get

$$
a \leq \frac{N^{-1 / p}}{\sqrt{\pi}}\left(\frac{p-1}{p-N}\right)^{1-1 / p}\left(\Gamma\left(1+\frac{N}{2}\right)\right)^{1 / N} m(\Omega)^{1 / N-1 / p},
$$

with $\Gamma$ being the gamma function. Since, for every $u \in W_{0}^{1, p}(\Omega)$,

$$
\|u\|_{p} \leq m(\Omega)^{1 / p}\|u\|_{\infty} \leq m(\Omega)^{1 / p} a\|u\|,
$$

Remark 2.1 provides

$$
b \leq \lambda_{1}^{-1 / p} \leq m(\Omega)^{1 / p} a \leq \frac{N^{-1 / p}}{\sqrt{\pi}}\left(\frac{p-1}{p-N}\right)^{1-1 / p}\left(m(\Omega) \Gamma\left(1+\frac{N}{2}\right)\right)^{1 / N} .
$$

Remark 3.5. Condition (3.3), with $c_{2}<\lambda_{1}$, appears also in [7, Proposition 4.1]. It is a simple matter to realize that this result and Corollary 3.3 are mutually independent.

REMARK 3.6. The main difficulty in treating the case $\Omega:=\mathbb{R}^{N}$ is to verify $\left(i_{1}\right)$. However, if the operator $A: W^{1, p}\left(\mathbb{R}^{N}\right) \rightarrow W^{-1, p^{\prime}}\left(\mathbb{R}^{N}\right)$ given by

$$
\langle A(u), v\rangle:=\int_{\mathbb{R}^{N}}\left(|\nabla u|^{p-2} \nabla u \cdot \nabla v+c(x)|u|^{p-2} u v\right) d x \quad \forall u, v \in W^{1, p}\left(\mathbb{R}^{N}\right),
$$

where $c \in L^{\infty}\left(\mathbb{R}^{N}\right)$ and ess $\inf _{x \in \Omega} c(x)>0$, takes the place of the one defined in (2.3), it can be done, as we shall see in a future work.

\section{References}

[1] D. Averna and S. A. Marano, 'Existence theorems for inclusions of the type $\Psi(u)(t) \in$ $F(t, \Phi(u)(t))^{\prime}$, Appl. Anal. 72 (1999), 449-458.

[2] D. Averna, S. A. Marano and D. Motreanu, 'Multiple solutions for a Dirichlet problem with $p$ Laplacian and set-valued nonlinearity', Bull. Aust. Math. Soc. 77 (2008), 285-303.

[3] G. Bonanno and G. Molica Bisci, 'Infinitely many solutions for a Dirichlet problem involving the p-Laplacian', Proc. Roy. Soc. Edinburgh Sect. A 140 (2010), 737-752.

[4] S. M. Bouguima, 'A quasilinear elliptic problem with a discontinuous nonlinearity', Nonlinear Anal. 25 (1995), 1115-1122.

[5] H. Brézis, Analyse Fonctionnelle-Théorie et Applications (Masson, Paris, 1983).

[6] V. I. Burenkov and V. A. Gusakov, 'On precise constants in Sobolev imbedding theorems', Sov. Math. Dokl. 35 (1987), 651-655.

[7] S. Carl and S. Heikkilä, ' $p$-Laplacian inclusions via fixed points for multifunctions in posets', Set-Valued Anal. 16 (2008), 637-649.

[8] S. Carl, V. K. Le and D. Motreanu, Nonsmooth Variational Problems and Their Inequalities (Springer, New York, 2007).

[9] J. Chabrowski, Variational Methods for Potential Operator Equations, de Gruyter Series in Nonlinear Analysis and Applications, 24 (de Gruyter, Berlin, 1997).

[10] L. Gasiński and N. S. Papageorgiou, Nonsmooth Critical Point Theory and Nonlinear Boundary Value Problems, Series in Mathematical Analysis and Applications, 8 (Chapman and Hall/CRC Press, Boca Raton, 2005). 
[11] P. Lindqvist, 'On the equation $\operatorname{div}\left(|\nabla u|^{p-2} \nabla u\right)+\lambda|u|^{p-2} u=0$ ', Proc. Amer. Math. Soc. 109 (1990), 157-164, Addendum: Proc. Amer. Math. Soc. 116 (1992), 583-584.

[12] R. Livrea and S. A. Marano, 'Non-smooth critical point theory', in: Handbook of Nonconvex Analysis and Applications (eds. D. Y. Gao and D. Motreanu) (International Press of Boston, Somerville, 2010), pp. 353-407.

[13] S. A. Marano, 'Existence theorems for a semilinear elliptic boundary value problem', Ann. Polon. Math. 60 (1994), 57-67.

[14] S. A. Marano and N. S. Papageorgiou, 'On some elliptic hemivariational and variationalhemivariational inequalities', Nonlinear Anal. 62 (2005), 757-774.

[15] I. Peral, 'Multiplicity of solutions for the $p$-Laplacian', in: ICTP Lecture Notes of the Second School of Nonlinear Functional Analysis and Applications to Differential Equations, Trieste, 1997.

[16] G. Talenti, 'Some inequalities of Sobolev type on two-dimensional spheres', in: General Inequalities 5, International Series of Numerical Mathematics, 80 (ed. W. Walter) (Birkhäuser, Basel, 1987), pp. 401-408.

S. A. MARANO, Dipartimento di Matematica e Informatica, Università degli Studi di Catania, Viale A. Doria 6, 95125 Catania, Italy e-mail: marano@dmi.unict.it 